\title{
RARE CARDIAC MASS ASSESSED BY CARDIAC MAGNETIC RESONANCE
}

\author{
Sebastian Militaru, Bernhard Gerber \\ Division of Cardiology, Department of Cardiovascular Diseases, \\ Cliniques Universitaires St. Luc \\ Pôle de Recherche Cardiovasculaire (CARD) \\ Institut de Recherche Expérimentale et Clinique (IREC) \\ Université Catholique de Louvain (UCLouvain), Brussels, Belgium
}

Keywords: Erdheim-Chester disease, cardiac mass, cardiac magnetic resonance

A 43 year old male was referred to our center for assessment of the cardiac involvement in Erdheim-Chester disease (EHD) by cardiac magnetic resonance (CMR). The patient presented with a history of bone involvement as well as retroperitoneal mass, demonstrated to consist fibrosis as well as histiocyte infiltration.

The CMR examination included cine (SSFP - steady state free procession), T1 weighted (T1w) and $\mathrm{T} 2$ weighted $(\mathrm{T} 2 \mathrm{w})$ sequences, as well as late enhancement images 10 minutes after gadolinium based contrast injection $(0.2 \mathrm{mmol} / \mathrm{kg})$. The acquired images showed normal dimensions and function for both right and left cardiac chambers. However, a cardiac mass was revealed in the free wall of the right atrium and the junction with the right ventricle, with clear borders and a diameter of $2.5 \mathrm{~cm}$ (Image 1). The tissue was best viewed on axial sequences and was isointense on cine, T1w and T2w images and was mildly enhanced on LGE images. Consequently, the diagnosis of cardiac involvement in EHD was confirmed. The patient was started on specific treatment for EHD and 3-year CMR follow-up showed regression of cardiac involvement.

Erdheim-Chester disease is a rare disorder most frequently characterized by non-Langerhans histiocytic multifocal osteosclerotic lesions, with multisystemic granulomatosis and widespread manifestations, as well as highly variable severity ${ }^{(1)}$. ECD affects the cardiovascular system in $75 \%$ of patients with infiltration of the pericardium and the right atrioventricular septum being the most common presentation. Typically the mass appears isointense on $\mathrm{Tl}$ and T2 weighted images and has low contrast enhancement, as was the case in our patient. In approximately $60 \%$ of cases death occurs because of cardiac complications, like pericardial tamponade, myocardial infarction, cardiomyopathy or arrhythmias ${ }^{(2)}$. Patients may sometimes be successfully treated with biologic therapy, interferon alpha or radiotherapy. 


\section{INTERNAL MEDI}

Images in Clinical'Medicine

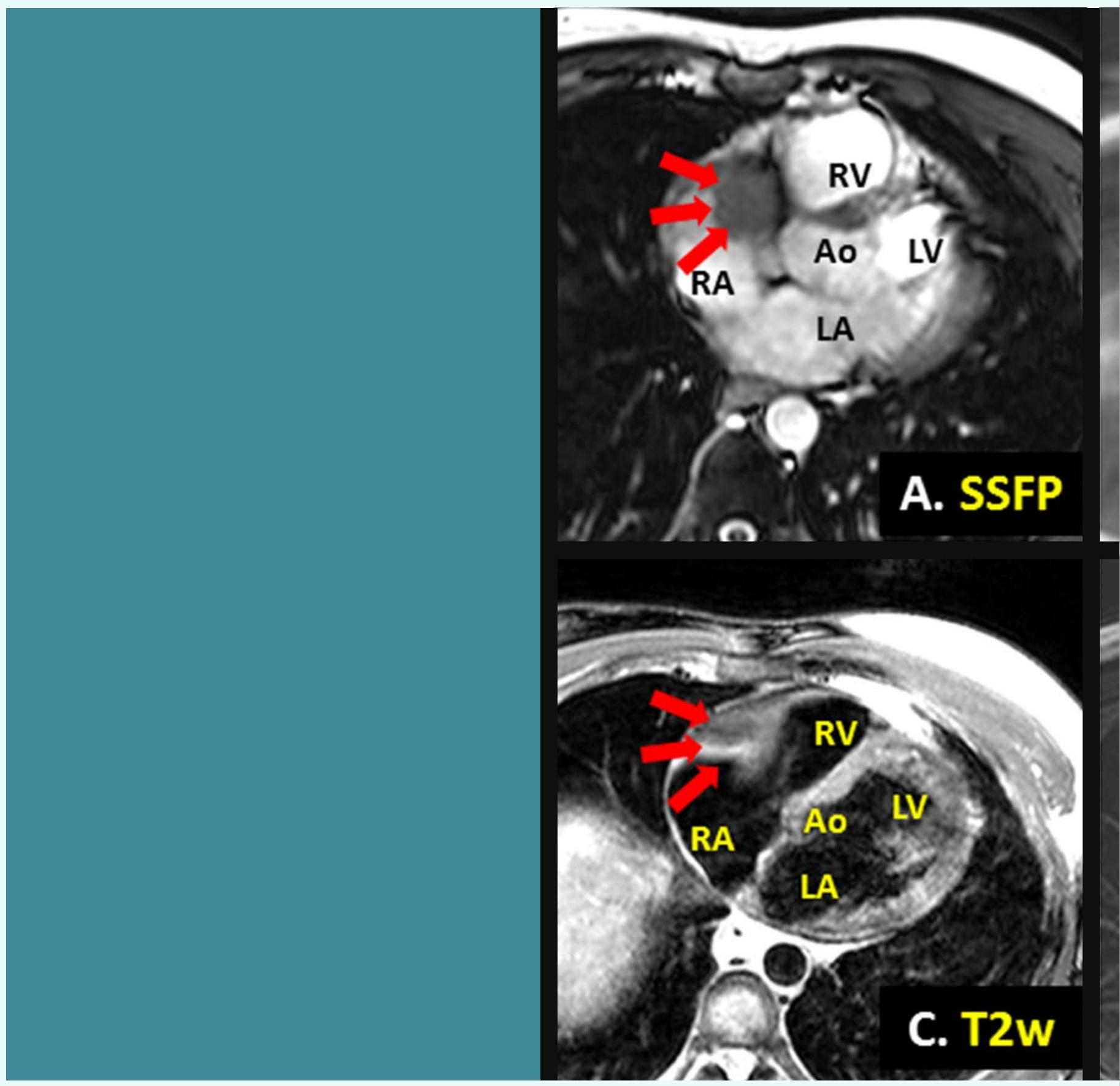


